\title{
The dynamics of a triopoly Cournot game when the competitors operate under capacity constraints
}

\author{
Tönu Puu ${ }^{a}$, Manuel Ruíz Marín ${ }^{b}$ \\ ${ }^{a}$ Centre for Regional Science, Umeå University, SE-90187 Umeå, Sweden \\ ${ }^{b}$ Facultad de C.C. de la Empresa, \\ Dpto. Métodos Cuantitativos e Informáticos, \\ Universidad Politécnica de Cartagena, \\ Paseo Alfonso XIII, 50 - 30.203, Cartagena, Spain
}

\section{Introduction}

Oligopoly theory, i.e., the economic theory for competition among the few, goes back to 1838 and Augustin Cournot [7]. See also [11]. Quite early it was suspected to lead to complex dynamic behaviour and chaos. See Rand 1978 [13]. The probably simplest case under which this happens with reasonable economics assumptions was suggested by one of the present authors in 1991, see [9]. It assumes an isoelastic demand function, which always arises when the consumers maximize utility functions of the Cobb-Douglas type, combined with constant marginal costs. The particular layout was a duopoly, the case of only two competitors. The model was shown to produce a period doubling sequence of flip bifurcations ending in chaos for the outputs of each of the two competitors.

Later the triopoly case under these assumptions was studied. See [2], [3], and [4] for examples. An interesting fact is that with three competitors the main frame becomes the Neimark-Hopf bifurcation, which provides new and different scenarios.

The main reason for economists to study increasing numbers of competitors is to find out whether it is the number of competitors that uniquely decides a road from monopoly over duopoly, oligopoly, and polypoly, to perfect competition, a state where each firm is so small that its actions cannot influence the market at all. To find out about this it is of primary interest to know whether the number of competitors stabilizes or destabilizes the equilibrium state. Some authors have questioned the assumption, to which most economists adhered, that increasing numbers of competitors bring stabilization.

However, we must be clear about what we compare. If we study increasing numbers of competitors with constant unit production costs, we are not reducing the size of the firms when their number increases. Constant marginal cost means that potentially each firm has infinite capacity, and adding such firms is not what we want for comparison.

It is therefore interesting to combine an increased number of firms with decreasing size of each firm, but in order to do so we have to introduce capacity limits. Already Edgeworth [8] insisted on the importance of capacity limits. It is not so easy to find non-constant marginal cost functions which allow us to solve for the reaction functions for the firms in explicit form, but one of the present authors, see [12], found one type of function, which models the capacity limit by letting marginal cost go to infinity at a finite output. That paper discussed the competition between two duopolists. The objective of the present paper is to find out the facts when there are three competitors, and we still keep the assumption of capacity limits.

\section{The model}

Consider three competitors whose supplies are denoted $x, y, z$. Further, assume an isoelastic demand function with the inverse, 


$$
p:=\frac{1}{x+y+z}
$$

that is market price is reciprocal to the sum of the three supplies.

Also assume cost functions of the shapes $T C_{1}(x):=-\log (u-x), T C_{2}(y):=-\log (v-y)$, and $T C_{3}(z):=$ $-\log (w-z)$. The parameters $u, v$, and $w$ denote capacity limits. The corresponding marginal cost functions then become $M C_{1}(x)=1 /(u-x), M C_{2}(y)=1 /(v-y)$, and $M C_{3}(z)=1 /(w-z)$ respectively. Observe that only the regions $x<u, y<v$, and $z<w$ are relevant. Obviously marginal costs, starting off modestly at zero output, go to infinity once the capacity limits are approached. This is as suggested by Edgeworth, though the capacity limits are approached asymptotically through rising costs.

As mentioned, one of the present authors [12], treated this case with two competitors in a previous publication, but interesting new phenomena occur when the number of competitors is increased. For instance, we get Neimark bifurcations, in stead of flip bifurcations, even without introducing any adaptation mechanism at all. This will be the case even if we make two of the competitors identical, for instance putting $v=w$, in which case the essentially two dimensional process takes place in the invariant plane $y=z$.

We are now able to state the profit expressions for the three competitors, $\Pi_{1}=p x-T C_{1}(x), \Pi_{2}=$ $p y-T C_{2}(y)$, and $\Pi_{3}=p z-T C_{3}(z)$

$$
\begin{aligned}
& \Pi_{1}(x, y, x)=\frac{x}{x+y+z}+\log (u-x), \\
& \Pi_{2}(x, y, x)=\frac{y}{x+y+z}+\log (v-y), \\
& \Pi_{3}(x, y, x)=\frac{z}{x+y+z}+\log (w-z) .
\end{aligned}
$$

Recall that throughout the paper we assume $x<u, y<v, z<w$. Then, maximizing profits, i.e., putting $\partial \Pi_{1} / \partial x=0, \partial \Pi_{2} / \partial y=0$, and $\partial \Pi_{3} / \partial z=0$, we can solve for the so called reaction functions $x=r_{1}(y, z)$, $y=r_{2}(x, z), z=r_{3}(x, y)$ for the three competitors, where

$$
\begin{aligned}
& r_{1}(y, z)=\frac{1}{2} \sqrt{4 u(y+z)+5(y+z)^{2}}-\frac{3}{2}(y+z) \\
& r_{2}(x, z)=\frac{1}{2} \sqrt{4 v(x+z)+5(x+z)^{2}}-\frac{3}{2}(x+z) \\
& r_{3}(x, y)=\frac{1}{2} \sqrt{4 w(x+y)+5(x+y)^{2}}-\frac{3}{2}(x+y)
\end{aligned}
$$

Now, assume the players move simultaneously at each stage of the game, using their reaction functions. We then have a three dimensional (3D henceforth) discrete model

$$
T=\left\{\begin{array}{l}
x^{\prime}=\frac{1}{2} \sqrt{4 u(y+z)+5(y+z)^{2}}-\frac{3}{2}(y+z) \\
y^{\prime}=\frac{1}{2} \sqrt{4 v(x+z)+5(x+z)^{2}}-\frac{3}{2}(x+z) \\
z^{\prime}=\frac{1}{2} \sqrt{4 w(x+y)+5(x+y)^{2}}-\frac{3}{2}(x+y)
\end{array}\right.
$$

As we want real iterates for the map $T$, which contains a square root, it must be constrained to a domain where the expression under the square root is positive. Then we have to ensure that $4 u(y+z)+5(y+z)^{2} \geq 0$, $4 v(x+z)+5(x+z)^{2} \geq 0$, and $4 w(x+y)+5(x+y)^{2} \geq 0$. Hence, there is a natural domain

$$
\begin{gathered}
D=\{x<u, y<v, z<w\} \cup\{(y+z) \geq 0,(x+z) \geq 0,(x+y) \geq 0\} \cup \\
\left\{(y+z) \leq-\frac{4}{5} u,(x+z) \leq-\frac{4}{5} v,(x+y) \leq-\frac{4}{5} w\right\}
\end{gathered}
$$

and we want to ensure that all the forward iterates $T^{n}(x, y, z)$ are contained in this set $D$. Otherwise, we could not compute the entire forward orbits in the set of real numbers. Following [3] and [4] we call the set of all the initial points for which this holds 


$$
S=\left\{(x, y, z) \in D \mid T^{n}(x, y, z) \in D \text { for all } n \geq 0\right\}
$$

the admissible set of points.

Further, in order to make sense in terms of economics, the map $T$ must be even more restricted. Outputs also have to be positive, so we consider a lower set $F \subseteq S \subseteq D$ of initial conditions for which all the forward iterates remain not only real numbers, but positive real numbers. This is called the feasible set, and is defined:

$$
F=\left\{(x, y, z) \in S \mid T^{n}(x, y, z) \in S \cap \mathbb{R}_{+}^{3} \text { for all } n \geq 0\right\}
$$

Unlike the case dealt with in [3] and [4], we now also have the capacity constraints to take in consideration, so we may want to substitute the box

$$
E=\{0 \leq x<u, 0 \leq y<v, 0 \leq z<w\},
$$

where $E \subset \mathbb{R}_{+}^{3}$, for $\mathbb{R}_{+}^{3}$ in the definition for $F$, but it makes no difference. We easily find from the definition of $T$ that in each application $0 \leq x<u, 0 \leq y<v$, and $0 \leq z<w$ are always automatically fulfilled.

There is another way to avoid all these problems, and that is to prescribe that all the entries in the map $T$, as defined above, are the maxima of the expressions stated and zero. In this way, used in the numerical studies below, all negative outputs are avoided. This makes perfect economic sense, as the implication is that if a firm cannot make any positive profit from production, it just stays idle and supplies nothing. Of course, the map then becomes only piecewise smooth. However, if we want to study which trajectories are feasible using only the regular definition in the map $T$, then the restriction to the set $F$ applies.

\section{The Cournot point $(\bar{x}, \bar{y}, \bar{z})$}

The Cournot equilibrium point is defined as the point where $x=r_{1}(y, z), y=r_{2}(x, z), z=r_{3}(x, y)$ hold as a simultaneous system of equations. It is not possible to get any nice closed form solution for the coordinates $\bar{x}, \bar{y}, \bar{z}$ of the Cournot equilibrium point in terms of the parameters $u, v, w$. However, we can treat $\bar{x}, \bar{y}, \bar{z}$ as parameters and solve for $u, v, w$. It will be seen that any combination of positive $\bar{x}, \bar{y}, \bar{z}$ is possible for a Cournot point. In the Cournot point we have that

$$
\begin{aligned}
u & =\frac{\bar{x}^{2}}{\bar{y}+\bar{z}}+3 \bar{x}+\bar{y}+\bar{z} \\
v & =\frac{\bar{y}^{2}}{\bar{x}+\bar{z}}+\bar{x}+3 \bar{y}+\bar{z} \\
w & =\frac{\bar{z}^{2}}{\bar{x}+\bar{y}}+\bar{x}+\bar{y}+3 \bar{z}
\end{aligned}
$$

Capacity limits are thus automatically fulfilled for $\bar{x}, \bar{y}, \bar{z} \geq 0$. Note that with nonnegative $\bar{x}, \bar{y}, \bar{z}$, the sum of all outputs is less than each of the capacity limits. Hence each component itself satisfies the proper capacity limit stated in its cost function. Also note that, when $\bar{x} \rightarrow 0$, then $\bar{y}+\bar{z} \rightarrow u$, when $\bar{y} \rightarrow 0$, then $\bar{x}+\bar{z} \rightarrow v$, and when $\bar{z} \rightarrow 0$, then $\bar{x}+\bar{y} \rightarrow w$

Further, the point $(0,0,0)$ satisfies equation 3.1. However the origin is not a feasible point. Moreover, it is easy to see that the function $T$ is not differentiable in this point, and a simple view of the Jacobian matrix of $T$ given by

$$
J(x, y, z)=\left(\begin{array}{ccc}
0 & \frac{2 u+5(y+z)}{2 \sqrt{(y+z)(4 u+5(y+z))}-\frac{3}{2}} & \frac{2 u+5(y+z)}{2 \sqrt{(y+z)(4 u+5(y+z))}-\frac{3}{2}} \\
\frac{2 v+5(x+z)}{2 \sqrt{(x+z)(4 v+5(x+z))}}-\frac{3}{2} & 0 & \frac{2 v+5(x+z)}{2 \sqrt{(x+z)(4 v+5(x+z))}-\frac{3}{2}} \\
\frac{2 w+5(x+y)}{2 \sqrt{(x+y)(4 w+5(x+y))}}-\frac{3}{2} & \frac{2 w+5(x+y)}{2 \sqrt{(x+y)(4 w+5(x+y))}}-\frac{3}{2} & 0
\end{array}\right)
$$


shows that the eigenvalues of $J$ tend to infinity as $(x, y, z)$ tends to the origin. Thus we may consider the origin as an unstable fixed point of the map $T$.

More difficult is to determine the stability conditions for the feasible fixed point $E^{*}=(\bar{x}, \bar{y}, \bar{z})$.

We can now substitute for $u, v, w$ in terms of $\bar{x}, \bar{y}, \bar{z}$, so reversing the role of variables and parameters, and obtain

$$
J^{*}=J(\bar{x}, \bar{y}, \bar{z})=\left(\begin{array}{ccc}
0 & A & A \\
B & 0 & B \\
C & C & 0
\end{array}\right)
$$

and $\operatorname{Det}\left(J^{*}\right)=2 A \cdot B \cdot C$ where

$$
\begin{aligned}
A & =\frac{\bar{x}^{2}-(\bar{y}+\bar{z})^{2}}{2 \bar{x}(\bar{y}+\bar{z})+3(\bar{y}+\bar{z})^{2}} \\
B & =\frac{\bar{y}^{2}-(\bar{x}+\bar{z})^{2}}{2 \bar{y}(\bar{x}+\bar{z})+3(\bar{x}+\bar{z})^{2}} \\
C & =\frac{\bar{z}^{2}-(\bar{x}+\bar{y})^{2}}{2 \bar{z}(\bar{x}+\bar{y})+3(\bar{x}+\bar{y})^{2}}
\end{aligned}
$$

Notice that the expressions of $A, B$ and $C$ only depend on $\frac{\bar{y}}{\bar{x}}=n$ and $\frac{\bar{z}}{\bar{x}}=m$. Indeed we have that

$$
\begin{aligned}
A & =\frac{1-(m+n)^{2}}{(m+n)(2+3 m+3 n)} \\
B & =\frac{n^{2}-(m+1)^{2}}{(m+1)(3(m+1)+2 n)} \\
C & =\frac{m^{2}-(n+1)^{2}}{(n+1)(3(n+1)+2 m)}
\end{aligned}
$$

The characteristic polynomial is

$$
\operatorname{Det}\left(J^{*}-\lambda I\right)=p_{3}(\lambda)=-\lambda^{3}+(A \cdot B+A \cdot C+B \cdot C) \lambda+2 A \cdot B \cdot C
$$

In order to check out the existence of Neimark bifurcations we need to know when the polynomial $p_{3}(\lambda)$ has two complex roots in the unit circle. We obtain the following result.

Proposition 3.1 Let $p(x)=-x^{3}+a x+b$ be a polynomial with coefficients $a, b \in \mathbb{R}$. Then $p(x)$ has two complex conjugated roots $\alpha_{1}, \alpha_{2} \in \mathbb{C}$ with $\left|\alpha_{1}\right|=\left|\alpha_{2}\right|=1$ if and only if $a=b^{2}-1$ and $|b|<2$.

\section{Proof.}

Let us now prove the $(\Rightarrow)$ part. Let $\alpha_{1}, \alpha_{2}, \alpha_{3}$ be the three roots of $p(x)$. Assume that $\alpha_{1}=s+i t$, $\alpha_{2}=s-i t$. Since $p(x)$ has 0 as the coefficient for the $x^{2}$ term, it follows that $\alpha_{1}+\alpha_{2}+\alpha_{3}=0$, and hence $\alpha_{3}=-2 s$. Since $\left|\alpha_{1}\right|=\left|\alpha_{2}\right|=1$ we have $\alpha_{1} \alpha_{2}=1$. Then, by Cardano's formulas, we have $b=\alpha_{1} \alpha_{2} \alpha_{3}=\alpha_{3}=-2 s$ and $a=-\left(\alpha_{1} \alpha_{2}+\alpha_{1} \alpha_{3}+\alpha_{2} \alpha_{3}\right)=-\left(1+\alpha_{3}\left(\alpha_{1}+\alpha_{2}\right)\right)=4 s^{2}-1$. Therefore $a=b^{2}-1$. Also, since $b=-2 s$ and $s=\operatorname{Re}\left(\alpha_{i}\right)$ and $\left|\alpha_{i}\right|=1, i=1,2$, it follows that $|b|<2$ which finishes the proof of the if part.

Now we prove the $(\Leftarrow)$ part. Assume that $p(x)=-x^{3}+\left(b^{2}-1\right) x+b$ with $|b|<2$. Then the roots of this polynomial are $\alpha_{1}=b, \alpha_{2}=-\frac{1}{2}\left(b+\sqrt{b^{2}-4}\right)$ and $\alpha_{3}=\frac{1}{2}\left(-b+\sqrt{b^{2}-4}\right)$. Since $|b|<2$ we have that $\alpha_{2}$ and $\alpha_{3}$ are complex conjugated roots and a straightforward computation shows that $\left|\alpha_{2}\right|=\left|\alpha_{3}\right|=1$.

In the case of $p_{3}(\lambda)$, it follows that it has two complex roots in the unit circle if the following equation is satisfied

$$
4 A^{2} B^{2} C^{2}-A B-A C-B C-1=0
$$

\section{Critical surfaces}

It is easy to see that our map is noninvertible, i.e., even if one point $(x, y, x) \in S$ is uniquely mapped into a point $\left(x^{\prime}, y^{\prime}, z^{\prime}\right)=T(x, y, z)$, the rank 1 preimage of a point $\left(x^{\prime}, y^{\prime}, z^{\prime}\right)$, belonging to $S$ may not exist, or may be a set of a finite number of distinct points. We recall that the critical points of rank 0 are points in which 
the Jacobian matrix of $T$ vanishes and the map is not locally invertible. The sets of such a points are called critical surfaces $(C S)$, of rank 0 , and are denoted $C S_{-1}$. From the Jacobian matrix given above we have

$\operatorname{Det}(J(x, y, z))=\frac{1}{8}\left(\frac{2 u+5(y+z)}{\sqrt{(y+z)(4 u+5(y+z))}}-3\right)\left(\frac{2 v+5(x+z)}{\sqrt{(x+z)(4 v+5(x+z))}}-3\right)\left(\frac{2 w+5(x+y)}{\sqrt{(x+y)(4 w+5(x+y))}}-3\right)$

so that the locus $\operatorname{Det}(J(x, y, z))=0$ is made up of six planes, portions of which constitute our critical set $C S_{-1}$ of rank 0 , which is made up of the intersection of the domain of $T$ with the planes given by equations: $y+z=\frac{u}{5}, x+z=\frac{v}{5}, x+y=\frac{w}{5}$, and $y+z=-u, x+z=-v, x+y=-w$.

As we are only interested in the region of feasible points $F$, in order to distinguish the different components of the critical sets we shall call

$$
\begin{gathered}
C S_{-1}^{(u)}=F \cap\left\{y+z=\frac{u}{5}\right\} \\
C S_{-1}^{(v)}=F \cap\left\{x+z=\frac{v}{5}\right\} \\
C S_{-1}^{(w)}=F \cap\left\{x+y=\frac{w}{5}\right\}
\end{gathered}
$$

The image by $T$ of such a portions of planes are critical surfaces of rank 1 , say $C S=T\left(C S_{-1}\right)$, where we shall distinguish three surfaces $C S^{(l)}=T\left(C S_{-1}^{(l)}\right), l=u, v, w$. It is easy to see that these three surfaces are the union of portions of the planes

$$
\begin{aligned}
& C S^{(u)} \text { belongs to the plane }\left\{x=\frac{u}{5}\right\} \\
& C S^{(v)} \text { belongs to the plane }\left\{y=\frac{v}{5}\right\} \\
& C S^{(w)} \text { belongs to the plane }\left\{z=\frac{w}{5}\right\}
\end{aligned}
$$

We recall that $C S$ separates zones of the phase-space, the points of which have a different number of distinct rank 1 preimages, and that crossing through a critical surface, the number of rank 1 preimages changes by 2 or a multiple of 2 . In order to understand which are the preimages and how many, we have to solve the system

$$
\begin{aligned}
& x^{\prime}=\frac{1}{2}\left(\sqrt{4 u(y+z)+5(y+z)^{2}}-3(y+z)\right) \\
& y^{\prime}=\frac{1}{2}\left(\sqrt{4 u(x+z)+5(x+z)^{2}}-3(x+z)\right) \\
& z^{\prime}=\frac{1}{2}\left(\sqrt{4 u(x+y)+5(x+y)^{2}}-3(x+y)\right)
\end{aligned}
$$

Assume a given admissible point $\left(x^{\prime}, y^{\prime}, z^{\prime}\right)$, and let us search for the solution vectors $(x, y, z)$. To accomplish this, define $\alpha=y+z, \beta=x+z$ and $\gamma=x+y$. Then we have

$$
\begin{aligned}
& \alpha_{ \pm}=\frac{1}{2}\left(u-3 x^{\prime} \pm \sqrt{\left(u-5 x^{\prime}\right)\left(u-x^{\prime}\right)}\right) \\
& \beta_{ \pm}=\frac{1}{2}\left(v-3 y^{\prime} \pm \sqrt{\left(v-5 y^{\prime}\right)\left(v-y^{\prime}\right)}\right) \\
& \gamma_{ \pm}=\frac{1}{2}\left(w-3 z^{\prime} \pm \sqrt{\left(w-5 z^{\prime}\right)\left(w-z^{\prime}\right)}\right)
\end{aligned}
$$

Then in order to have $\left(u-5 x^{\prime}\right)\left(u-x^{\prime}\right) \geq 0$ (for the existence of $\alpha_{ \pm}$) we need that $x^{\prime} \leq \frac{u}{5}$ and then $\alpha_{ \pm} \geq 0$. By symmetry we obtain:

$$
\begin{array}{ll}
\alpha_{ \pm}=\frac{1}{2}\left(u-3 x^{\prime} \pm \sqrt{\left(u-5 x^{\prime}\right)\left(u-x^{\prime}\right)}\right) & \text { if } x^{\prime} \leq \frac{1}{5} u \\
\beta_{ \pm}=\frac{1}{2}\left(v-3 y^{\prime} \pm \sqrt{\left(v-5 y^{\prime}\right)\left(v-y^{\prime}\right)}\right) & \text { if } x^{\prime} \leq \frac{1}{5} v \\
\gamma_{ \pm}=\frac{1}{2}\left(w-3 z^{\prime} \pm \sqrt{\left(w-5 z^{\prime}\right)\left(w-z^{\prime}\right)}\right) & \text { if } z^{\prime} \leq \frac{1}{5} w
\end{array}
$$


Therefore, for a given tern $(\alpha, \beta, \gamma)$, from the relations above we get one of the inverses of $T$ which reads as

$$
T^{-1}\left(x^{\prime}, y^{\prime} z^{\prime}\right)=\left(\frac{1}{2}(-\alpha+\beta+\gamma), \frac{1}{2}(\alpha-\beta+\gamma), \frac{1}{2}(\alpha+\beta-\gamma)\right)
$$

Then we obtain several zones in the admissible region of the phase-space, as stated in the following proposition. The symbol $Z_{k}$ is used to denote a region whose points have $k$ distinct rank-1 preimages.

Proposition 4.1 Let $(x, y, z)$ be an admissible point. Then

a) $Z_{0}$ is the region of the space for which $\frac{1}{5} u<x<u$ or $\frac{1}{5} v<y<v$ or $\frac{1}{5} w<z<w$

b) $Z_{8}=D \cap\left(Z_{0}\right)^{c}$ where $\left(Z_{0}\right)^{c}$ means the complement of the region $Z_{0}$

\section{$5 \quad$ Topological conjugacy and symmetry properties}

That the three parameters of the map $T$ are not all independent from a dynamical point of view is due to the following proposition:

Proposition 5.1 The dynamics of the map $T$ in terms of parameters $(u, v, w)$ and $(\tau u, \tau v, \tau w)$ with $\tau>0$ are topologically conjugated via the homeomorphism $\phi(x, y, z)=(\tau x, \tau y, \tau z)$

We just need a straightforward computation to see that, if we change the three parameters $(u, v, w)$ to $(\tau u, \tau v, \tau w)$, with $\tau>0$, then we obtain a $3 D$ map, say $\widetilde{T}$, which, given $\widetilde{T}=\phi \circ T \circ \phi^{-1}$, and using the homeomorphism $\phi(x, y, z)=(\tau x, \tau y, \tau z)$, is topologically conjugated with $T$.

Note that the homeomorphism $\phi$ transforms admissible (respectively feasible) trajectories to admissible (respectively feasible) trajectories. If $S$ (respectively $F$ ) is the considered as the application set for $T$, then $\phi(S)$ (respectively $\phi(F)$ ) is the corresponding set of $\widetilde{T}$.

From this proposition it follows that the reduced parameters $\frac{v}{u}$ and $\frac{w}{u}$ of the map are the two essential independent parameters which we vary in order to investigate the dynamic behavior of the map $T$. This is so since the dynamics in the phase-space, associated with $(u, v, w)$, is topologically conjugated to those of the map $\widetilde{T}$ with the parameters $\left(1, \frac{v}{u}, \frac{w}{u}\right)$

Proposition 5.2 The dynamics of the map $T$ with terns of parameters $\left(1, \frac{v}{u}, \frac{w}{u}\right)$ and $\left(1, \frac{w}{u}, \frac{v}{u}\right)$ are topologically conjugated via the homeomorphism $\varphi(x, y, z)=(x, z, y)$

As a consequence of Proposition 5.2 we conclude that, in the reduced parameter plane, the bifurcation curves in the $\left(\frac{v}{u}, \frac{w}{u}\right)$ plane must be symmetric with respect to the bisectrix $\left(\frac{v}{u}=\frac{w}{u}\right)$.

From Proposition 5.2 we also have a symmetry property, which holds in the case of identical reduced parameters $\frac{v}{u}=\frac{w}{u}$. In this case a trajectory in phase-space is such that it either itself is symmetric with respect to the plane $y=z$, or else there exists another symmetric trajectory.

In particular, the plane with equation $y=z$ is invariant. Note that in terms of the three parameters $(u, v, w)$ of the map $T$ this case corresponds to identical parameters for the last two oligopolists, i.e., $v=w$. This is not the only case in which we have an invariant plane for the map. In fact, $x=y, x=z$, are invariant, provided that $u=v, u=w$ respectively.

In particular, the restriction of $T$ to an invariant plane can be identified with a $2 D$ map. The restrictions of $T$ to these three invariant planes are topologically conjugated. And also the dynamics of the $3 D$ map $T$ in such cases turns out to be conjugated.

These properties can be formulated in terms of terns of the original parameters as follows:

Proposition 5.3 (1) The dynamics of the map $T$ with terns of parameters $(u, v, w)$ and $(u, w, v)$ are topologically conjugated via the homeomorphism $\varphi_{1}(x, y, z)=(x, z, y)$, and in the case $v=w$ the plane $y=z$ is invariant. Moreover the trajectories are symmetric with respect the plane $y=z$ or symmetric trajectories exist. 
(2) The dynamics of the map $T$ with terns of parameters $(u, v, w)$ and $(w, v, u)$ are topologically conjugated via the homeomorphism $\varphi_{2}(x, y, z)=(z, y, x)$, and in the case $u=w$ the plane $x=z$ is invariant. Moreover the trajectories are symmetric with respect the plane $x=z$ or symmetric trajectories exist.

(3) The dynamics of the map $T$ with terns of parameters $(u, v, w)$ and $(v, u, w)$ are topologically conjugated via the homeomorphism $\varphi_{3}(x, y, z)=(y, x, z)$, and in the case $u=v$ the plane $x=y$ is invariant. Moreover the trajectories are symmetric with respect the plane $x=y$ or symmetric trajectories exist.

Finally we recall the similar properties coming from the rotations of the parameter values, which imply topological conjugacy in the phase-spaces by using rotations around the axis $x=y=z$

Proposition 5.4 (4) The dynamics of the map $T$ with terns of parameters $(u, v, w)$ and $(w, u, v)$ are topologically conjugated via the homeomorphism $\varphi(x, y, z)=(z, x, y)$

(5) The dynamics of the map $T$ with terns of parameters $(u, v, w)$ and $(v, w, u)$ are topologically conjugated via the homeomorphism $\varphi^{2}(x, y, z)=(y, z, x)$

These properties can also be deduced via a composition of two of the conjugacies given in Proposition 5.3 , i.e, $\varphi_{1} \circ \varphi_{2}(u, v, w)=\varphi_{2} \circ \varphi_{3}(u, v, w)=\varphi_{3} \circ \varphi_{1}(u, v, w)=(w, u, v)$. The homeomorphism $\varphi^{2}=\varphi_{1} \circ \varphi_{2}=$ $\varphi_{2} \circ \varphi_{3}=\varphi_{3} \circ \varphi_{1}$ is a rotation by $2 \pi / 3$ to the right of the oriented vector $(1,1,1)$.

Likewise $\varphi_{2} \circ \varphi_{1}(u, v, w)=\varphi_{3} \circ \varphi_{2}(u, v, w)=(v, w, u)$. The homeomorphism $\varphi^{2}=\varphi_{2} \circ \varphi_{1}=\varphi_{3} \circ \varphi_{2}=$ $\varphi_{1} \circ \varphi_{3}$ is a rotation by $4 \pi / 3$ to the right (or equivalently of $-2 \pi / 3$ to the left) of the oriented vector $(1,1,1)$.

\section{The dynamics of $T$ in the case $u=v$}

In this section we consider the particular case in which two of the parameters are equal. Due to the symmetry of the model, the dynamics of $T$ when two parameters of the model are equal, are conjugated, so let us assume $u=v$. This means that, if the initial values of $x$ and $y$ (outputs of the competitors), say $x_{0}$ and $y_{0}$, are equal, then the two players will move identically for ever, i.e., $x_{t}=y_{t}$ for any $t \geq 0$. If their initial states are not equal, then their trajectories will be different, but it is possible, or highly probable (depending on the structure of the basins), that ultimately their asymptotic behavior is similar.

Mathematically this comes from the fact that, assuming $u=v$, the plane $P^{*}$ of equation $x=y$ is trapping. It follows that the dynamics of points belonging to $P^{*}$ can be studied by the restriction of $T$ to the invariant plane $P^{*}$, which can be identified with a $2 D$-map. Let $a$ denote the common value $x=y$. Then the dynamics of $T$ on $P^{*}$ can be identified with the $2 D$-map $T_{a}$ given by:

$$
T_{a}=\left\{\begin{array}{l}
a^{\prime}=\frac{1}{2} \sqrt{4 u(a+z)+5(a+z)^{2}}-\frac{3}{2}(a+z) \\
z^{\prime}=\sqrt{2 w a+5 a^{2}}-3 a
\end{array}\right.
$$

Like in the $3 D$-map, it is not possible to get any nice closed form solution for the coordinates of the Cournot point for $T_{a}$, so, again reversing the roles of variables and parameters, we find that at the Cournot point $(\bar{a}, \bar{z})$ the parameters are:

$$
\begin{aligned}
u & =\frac{\bar{a}^{2}}{\bar{a}+\bar{z}}+4 \bar{a}+\bar{z} \\
w & =\frac{\bar{z}^{2}}{2 a}+2 a+3 \bar{z}
\end{aligned}
$$

Let us examine the stability of the Cournot point $E_{a}^{*}=(\bar{a}, \bar{z})$ The Jacobian matrix of $T_{a}$ is given by:

$$
J_{T_{a}}(u, z)=\left(\begin{array}{cc}
\frac{2 u+5(a+z)}{2 \sqrt{4 u(a+z)+5(a+z)^{2}}}-\frac{3}{2} & \frac{2 u+5(a+z)}{2 \sqrt{4 u(a+z)+5(a+z)^{2}}}-\frac{3}{2} \\
\frac{5 a+w}{\sqrt{2 w a+5 a^{2}}}-3 & 0
\end{array}\right)
$$


and its evaluation in the fixed point $E^{*}=(\bar{a}, \bar{z})$ gives:

$$
J_{T_{a}}(\bar{a}, \bar{z})=\left(\begin{array}{cc}
A & A \\
B & 0
\end{array}\right)
$$

where

$$
\begin{aligned}
& A=-\frac{\bar{z}(\bar{z}+2 \bar{a})}{(\bar{a}+\bar{z})(5 \bar{a}+3 \bar{z})} \\
& B=\frac{\bar{z}^{2}-4 \bar{a}^{2}}{2 \bar{a}(3 \bar{a}+\bar{z})}
\end{aligned}
$$

Note that the expressions $A$ and $B$ only depend on $\frac{\bar{z}}{\bar{a}}=m$. More concretely we have that

$$
\begin{aligned}
& A=-\frac{m(m+2)}{(m+1)(3 m+5)} \\
& B=\frac{(m+2)(m-2)}{2(m+3)}
\end{aligned}
$$

so that the characteristic polynomial becomes

$$
p_{2}(\lambda)=\lambda^{2}+\frac{m(m+2)}{(m+1)(3 m+5)} \lambda+\frac{m(m+2)^{2}(m-2)}{2(m+3)(m+1)(3 m+5)}
$$

The sufficient conditions for the local stability of the fixed point for the map $T_{a}$ are:

(1) $p_{2}(1)=\frac{30+62 m+40 m^{2}+10 m^{3}+m^{4}}{30+58 m+34 m^{2}+6 m^{3}}>0$, which is always satisfied for $m>0$,

(2) $p_{2}(-1)=\frac{30+38 m+20 m^{2}+6 m^{3}+m^{4}}{30+58 m+34 m^{2}+6 m^{3}}>0$, which is always satisfied for $m>0$,

(3) $\operatorname{Det}\left(J_{T_{a}}(\bar{a}, \bar{z})\right)=\frac{m(m+2)^{2}(m-2)}{2(m+3)(m+1)(3 m+5)}<1$, which is satisfied for $0<m<9.0471 \ldots$

Within this stability interval for the fixed point, we have in the middle an interval of parameter values to which there corresponds a stable node (i.e. the solutions of $p_{2}(\lambda)=0$ are real), while near the extremes of the stability interval, the solutions of $p_{2}(\lambda)=0$ are complex conjugated (so that the fixed point is a stable focus) approaching the modulus $1(0.999 \ldots)$. Thus the bifurcation values correspond to the Neimark-Hopf bifurcations of $E_{a}^{*}$. Let us first complete the local stability analysis for the fixed point for the map $T$ in the $3 D$ phase space by computing the third eigenvalue of the $3 D$ Jacobian matrix. The cubic polynomial defined in $(3.2)$ can be factorized as follows:

$$
p_{3}(\lambda)=p_{2}(\lambda)\left(\lambda-\lambda_{3}\right) \quad \lambda_{3}=\frac{m(m+2)}{(m+1)(5+3 m)}
$$

so the third eigenvalue of $T$ is always associated with a direction attracting towards the invariant plane, at least locally (near the fixed point). As $\lambda_{3}>0$, the trajectories are locally on one side of that plane, i.e., the trajectories are not oscillating from one side to the other, as is the case with a negative eigenvalue. This implies that the trajectories of points outside the invariant plane and near the fixed point cannot be symmetric with respect to the invariant plane.

Let us denote by $F_{a}$ the set of feasible points of $T_{a}$. Then the critical lines for the map $T_{a}$ are

$$
\begin{aligned}
& L C_{-1}^{(w)}=F_{a} \cap\left\{a=\frac{w}{10}\right\} \\
& L C_{-1}^{(u)}=F_{a} \cap\left\{a+z=\frac{u}{5}\right\}
\end{aligned}
$$

These lines correspond to the intersection of the critical surfaces $C S_{-1}$ with the invariant plane $P^{*}$. In fact, it is easy to see that

$$
\begin{aligned}
& L C_{-1}^{(w)}=C S_{-1}^{(w)} \cap P^{*} \\
& L C_{-1}^{(u)}=C S_{-1}^{(u)} \cap P^{*}=C S_{-1}^{(v)} \cap P^{*}
\end{aligned}
$$


But we can also say more about the attractivity of the invariant plane, because we can explicitly write the transverse eigenvalue for any admissible point $(a, a, z) \in P^{*}$. In fact from the Jacobian matrix of $T$ we have (for $u=v$ and $x=y=a$ )

$$
\lambda_{3}(a, a, z)=\frac{3}{2}-\frac{2 u+5(a+z)}{2 \sqrt{4 u(a+z)+5(a+z)^{2}}}
$$

Obviously, above the critical line $L C_{-1}^{(u)}$, the transverse eigenvalue is positive, whereas it is negative below it. Formally

$$
\lambda_{3}(a, a, z)>0 \Leftrightarrow a+z \geq \frac{1}{5} u
$$

so trajectories outside the invariant plane, far from the fixed point may also cross the plane whereas they cannot do this in the region above the critical line $L C_{-1}^{(u)}$. Moreover, $\left|\lambda_{3}(a, a, z)\right|<1$ is satisfied when $a+z \geq \frac{\sqrt{5}-2}{5} u$, so the transverse attractivity exists for a wide portion of $P^{*}$.

\section{Conclusion}

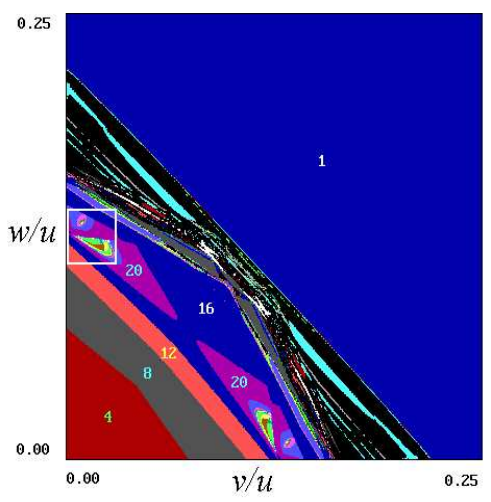

Figure 1: The bifurcation plane $v / u, w / u$.

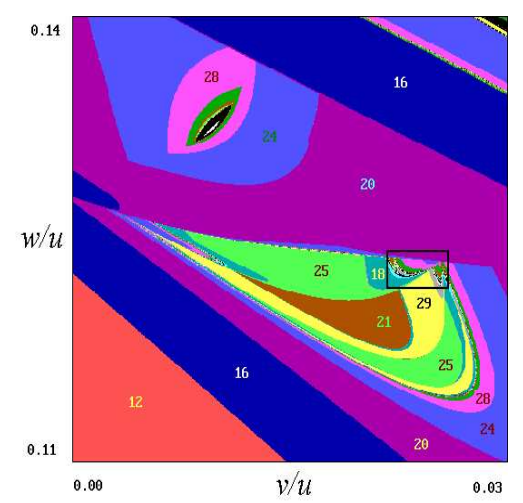

Figure 2: Enlarged picture of the rectangle in Figure 1.

Let us now finish this off by checking out the bifurcation plane numerically. Remember that, even though we in the general case have a three dimensional system, $x, y, z$ with three parameters $u, v, w$, there are only two free parameters that actually influence the behaviour of the system, the capacity ratios $v / u$ and $w / u$. 


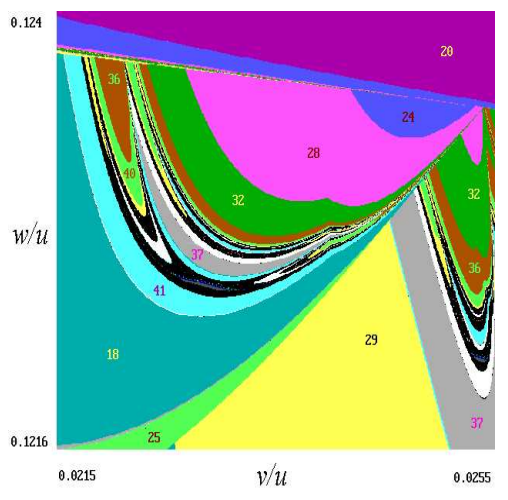

Figure 3: Further enlargement of the rectangle in Figure 2.

Hence we can easily produce two dimensional bifurcation diagrams and check out for instance the periodicity structure.

In Fig. 1 we see the interesting part of the bifurcation plane. Above the "negative diagonal", i.e., the bifurcation line, we find that the fixed point is stable. Proceeding along the positive diagonal, we see how 4-period orbits are replaced by 8-period, 12-period, and 16-period, always seemingly through a period adding progress of four. Complexity increases close to the bifurcation line before we enter the region of stable fixed point.

More interesting than this are the off diagonal asymmetric portions of the bifurcation plane, where complexity seems to increase. In Figs. 2 and 3 we take successive close up pictures of this region, indicated by boxes in the previous pictures, just to illustrate the very rich periodicity structure.

There obviously remain many numerical investigations to be made in order to understand the nature of bifurcations. We have focused on the local Neimark-Hopf bifurcations of the fixed point, but there seem to be interesting global bifurcations to study. As the fixed point bifurcation seems to be subcritiacal, such bifurcations give rise to coexistence of the fixed Cournot point and more complex attractors. (See [5] and [6] for some studies of very similar cases.)

\section{References}

[1] Abraham R, Gardini L, and Mira C. Chaos in Discrete Dynamical Systems: A Visual Introduction in 2 Dimensions. Springer-Verlag, 1997.

[2] Agiza HN. Explicit stability zones for Cournot games with 3 and 4 competitors. Chaos, Solitons \& Fractals, 1998; 9:1955-1966.

[3] Agliari A, Gardini L, and Puu T. The dynamics of a triopoly Cournot game. Chaos, Solitons \& Fractals, 2000; 11:2531-2560.

[4] Agliari A, Gardini L, and Puu T. Global bifurcations and basins in a triopoly game. International Journal of Bifurcations and Chaos, 2001; 12:2175-2207.

[5] Agliari A, Gardini L, and Puu T. Global bifurcations in duopoly when the Cournot Point is destabilized through a Subcritical Neimark bifurcation. International Game Theory Review, 2005 (in press)

[6] Agliari A, Gardini L, and Puu T. Some global bifurcations related to the apperance of closed invariant curves. Mathematics and Computers in Simulations, 2005 (in press)

[7] Cuornot A. Recherches sur les Principes Mathématiques de la Théorie des Richesses. Paris: Hachette, 1838.

[8] Edgeworth FY. La teoria pura del monopolio. Giornale degli Economisti, 1897; 15:13-31. 
[9] Puu T. Chaos in duopoly pricing. Chaos, Solitons \& Fractals, 1991; 1:573-581.

[10] Puu T. Attractors, Bifurcations \& Chaos - Nonlinear Perspectives in Economics. Springer-Verlag, 2003.

[11] Puu T and Suskho I. Oligopoly Dynamics - Models and Tools. Springer-Verlag, 2002.

[12] Puu T and Norin A. Cournot duopoly when the competitors operate under capacity constraints. Chaos, Solitons and Fractals, 2003; 18: 577-592

[13] Rand D. Exotic phenomena in games and duopoly models. Journal of Mathematical Economics, 1978; 5:173-184. 